\title{
Arrival-time analysis of intravenous digital aortograms in aortic dissection
}

\author{
J LYONS, A GERSHLICK, J GARDENER, C LAYTON
}

From the Department of Cardiology, London Chest Hospital, London

SUMMARY Arrival-time analysis was applied to intravenous digital aortograms. A single static image was produced by representing the time to $90 \%$ maximum density for each image pixel on a grey scale. Arrival-time analysis confirmed the diagnosis in four of six patients with aortic dissection that had been shown by image subtraction. In a further three patients with no dissection the arrival-time images were normal. In two patients with surgical repair of the ascending aorta, residual false lumens were shown by both subtraction and arrival-time analysis. In one of these cases arrival-time analysis prompted reanalysis of the subtraction image that had previously been interpreted as showing a normal aorta.

Arrival-time analysis provides a static image of aortic dissection, which helps to overcome subtraction artefact.

We have already shown the value of intravenous digital subtraction aortography in the diagnosis of acute aortic dissection. ${ }^{1}$ We found that this technique was as specific and sensitive as direct cineaortography. Careful interpretation of the images is essential to prevent overdiagnosis of dissection through misinterpretation of linear subtraction artefacts as being the intimal dissection flap, particularly when static images are studied. This problem can be overcome by acquiring images at a rapid frame rate and by viewing the whole sequence. This will detect the characteristic motion of the dissection flap throughout the cardiac cycle. Subtraction artefacts can be abolished by the development of analysis techniques that do not require image subtraction.

We have therefore developed computer software to produce arrival-time images of the aorta. These have the advantage of avoiding image subtraction, and thus permit an assessment of a complete time series of images in one static image. Used in combination with digital subtraction they should give more accurate diagnoses.

\section{Patients and methods}

To evaluate arrival-time imaging in the diagnosis of

Requests for reprints to Dr J Lyons, Department of Cardiology, London Chest Hospital, Bonner Road, London E2 9JX

Accepted for publication 8 September 1987 aortic dissection we reassessed several intravenous digital aortograms from our magnetic tape database. These included data from seven out of 11 patients who have been reported elsewhere ${ }^{1}$; data from the remaining four cases were not available on tape. All seven patients had presented with a clinical diagnosis of suspected acute aortic dissection. In three, digital subtraction had shown a dissection of the ascending aorta and this had been confirmed at operation. In one case the subtraction images had demonstrated dissection confined to the descending aorta, and this had been confirmed by cineangiography; operation was not undertaken. In three cases, no dissection had been seen either on the digital subtraction aortograms or at direct cineaortography.

We also analysed data from patients examined after the publication of our previous report. In two patients digital subtraction showed a chronic dissection of the ascending aorta, and this was confirmed by cineangiography in both cases and at operation in one. Postoperative data from two patients were also analysed by arrival-time processing. In one case the ascending aorta had been replaced and the investigation was performed to assess the extent of the residual dissection. A dissection extending from a point just distal to the left subclavian artery down to the aortic bifurcation was seen on the subtraction images. In the second case arrival-time analysis was performed at the time of the initial digital aortogram. The ascending aorta 
had been repaired with a sleeve of Dacron, and the digital investigation was performed because a small bulge was seen on the right side of the ascending aorta on a conventional chest $x$ ray.

Intravenous digital aortography was performed as described elsewhere. ${ }^{1}$ The digital system used was the Siemens Digitron 2 connected on-line to a standard Siemens Angioskop system. Digital aortograms were performed by pulsed radiography at 12.5 frames/second and displayed on a $256 \times 256$ matrix. Angiography was performed percutaneously via the antecubital or femoral vein with a 5 French Cordis Superflow pigtail catheter positioned in the right atrium to achieve a central injection of contrast medium. A $30^{\circ}$ or $60^{\circ}$ left anterior oblique projection was used; in some cases both projections were used if image quality and patient compliance were poor. Images were acquired over a 10 second period after a bolus injection of $40 \mathrm{ml}$ iohexol at $20 \mathrm{ml} / \mathrm{s}$ with a 5 s delay to allow for pulmonary transit.

The resultant images were then examined by arrival-time analysis as follows. The maximum and minimum densities throughout the image time series were determined for each image pixel. The time from the start of the series to $90 \%$ maximal density of each pixel (the arrival-time) was then determined (fig 1). A single static image was produced, each image pixel being represented on a grey scale according to its arrival-time-early arrival was light and late arrival was dark. The resultant images were then compared with the standard subtraction images to assist in the accurate diagnosis of aortic dissection.

\section{Results}

In four of the six patients with known dissection, the arrival-time images clearly demonstrated the true and false lumens by a difference in arrival-time (figs 2 and 3). All these dissections were in the ascending aorta and the delayed arrival that represented the false lumen was seen on the right-anterior aspect of the aorta in all cases. In two of these four cases a gradation of arrival-time was seen in the false lumen,

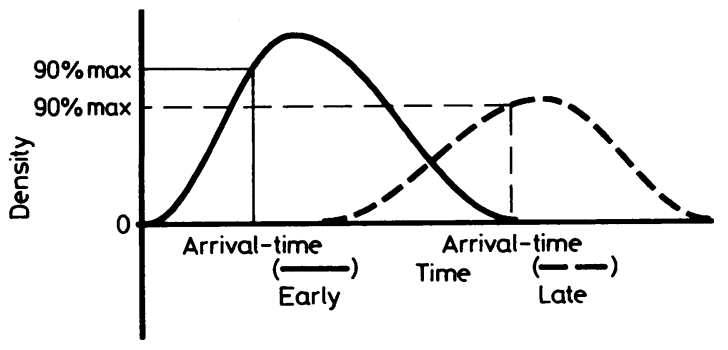

Fig 1 Time-density curves for two image pixels demonstrating the method of measuring arrival-time.
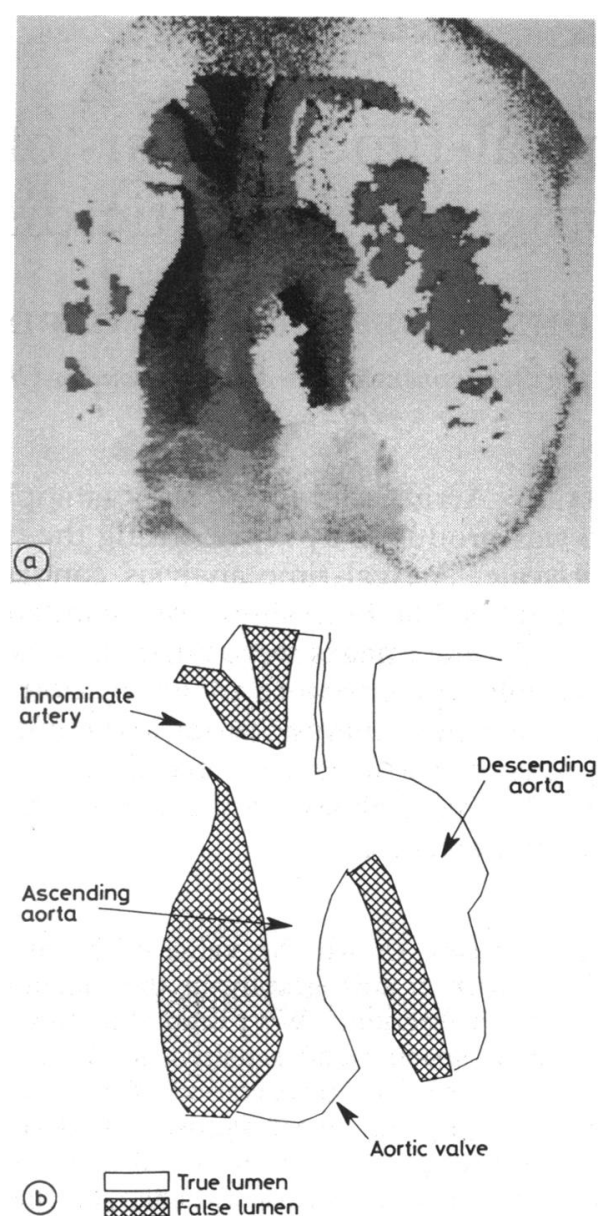

Fig 2 Arrival-time image (a) showing a dissection of the aorta extending from the aortic valve into the innominate artery and the descending aorta. (b) Explanatory diagram.

with the earliest arrival occurring just proximal to $\frac{7}{2}$ the innominate artery in one case and just distal to the left subclavian in the second case. At operation $N$ the entry site of the dissection was in the ascending $N$ aorta well above the coronary orifices in the first case and no entry site was found in the ascending aorta in $\omega_{-}^{\omega}$ the second case, indicating that it was distal to the left subclavian artery. In the other two cases arrival- - 을 time analysis showed that the false lumen extended into the innominate artery.

In two patients, one with dissection of the ascending aorta and the other with dissection of the descending aorta, no dissection was seen on the $\overrightarrow{\mathbb{D}}$ arrival-time images. When the subtraction images $\varrho$ were reviewed in these two cases multiple linear 

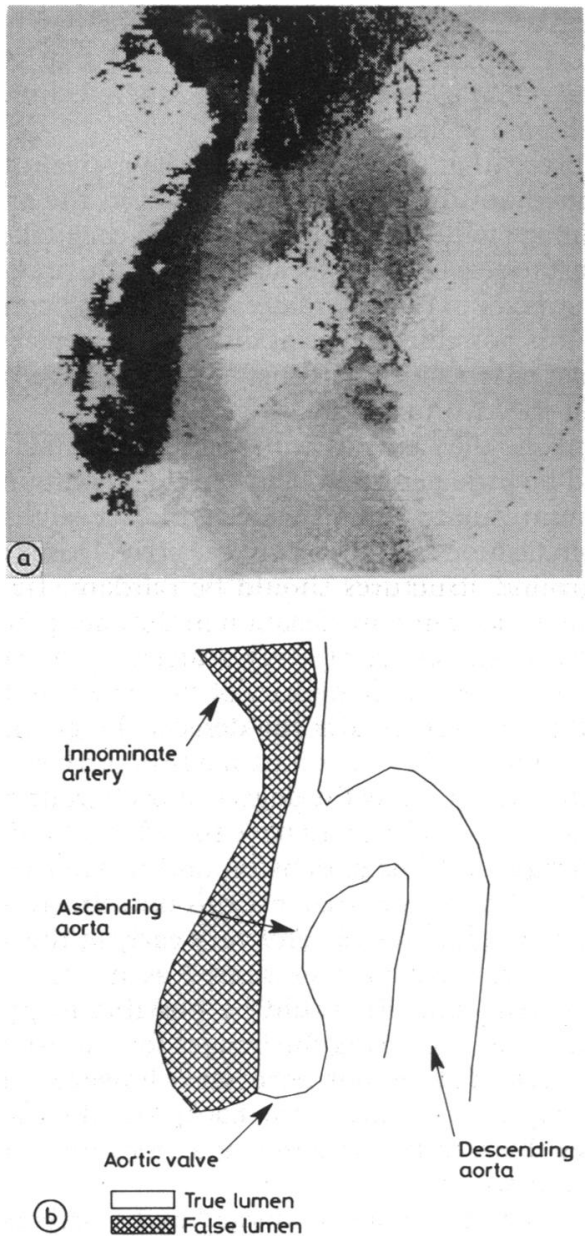

Fig 3 Arrival-time image (a) showing

dissection of the aorta extending from the aortic valve into the innominate artery. (b)

Explanatory diagram.

opacities were apparent, suggesting severe disruption of the intimal flap, such that the true and false lumens filled simultaneously.

In the three patients previously shown both by subtraction and by conventional cineaortography to have no dissection the arrival-time images demonstrated the expected pattern of contrast distribution throughout the aorta with a progressive delay in arrival-time throughout the length of the aorta.

Two patients were studied after operation. In the first the presence of a dissection extending from a point just distal to the left subclavian artery down to the aortic bifurcation was confirmed by the arrivaltime image in which the false lumen was clearly demonstrated by a delay in arrival-time (fig 4). The con-
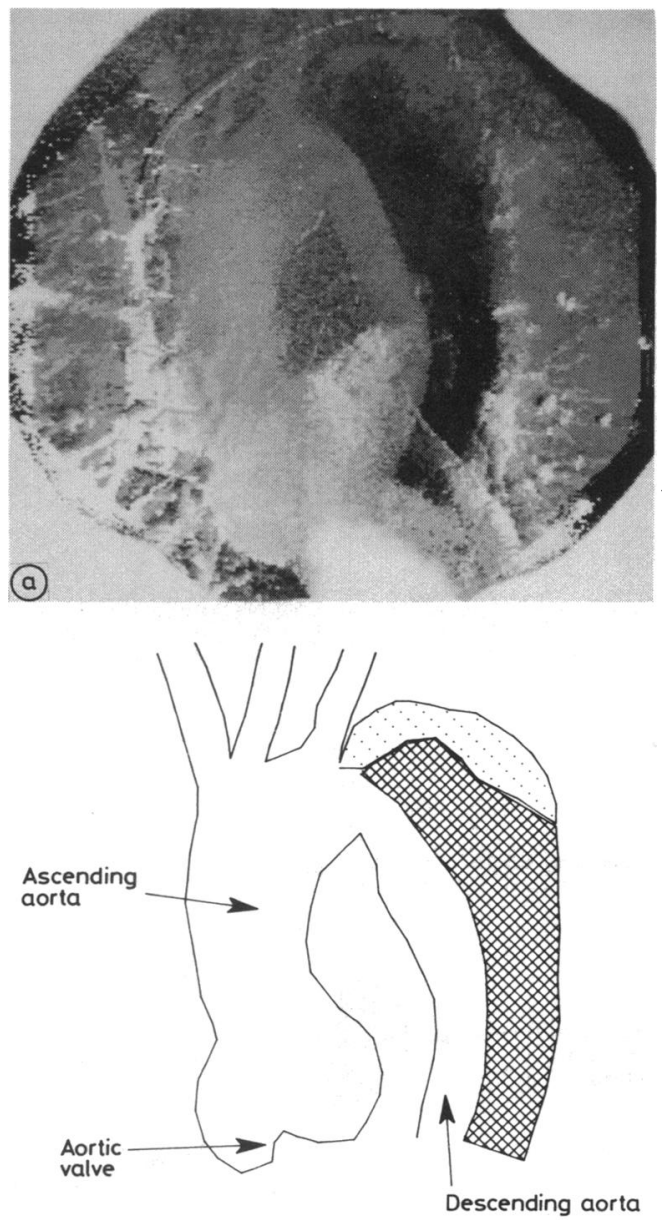

(b) $\begin{aligned} & \square \\ & \text { True lumen } \\ & \text { Entry site }\end{aligned}$

Fig 4 Arrival-time image (a) showing a residual false lumen in the descending aorta after repair of the ascending aorta. (b) Explanatory diagram. Early enhancement by contrast showed that the entry site of the false lumen was just below the left subclavian artery.

trast first arrived in the false lumen at the level of the left subclavian artery, with progressive delay in arrival-time along the length of the descending aorta. In the second patient digital investigation was performed to determine the nature of a small bulge on the right side of the aorta and the ascending aorta was initially thought to be normal on the subtraction images. There was a considerable delay in arrivaltime in a small area in the right-anterior aspect of the aorta, corresponding to the bulge on the chest $x$ ray, and this drew attention to a small leak of contrast 

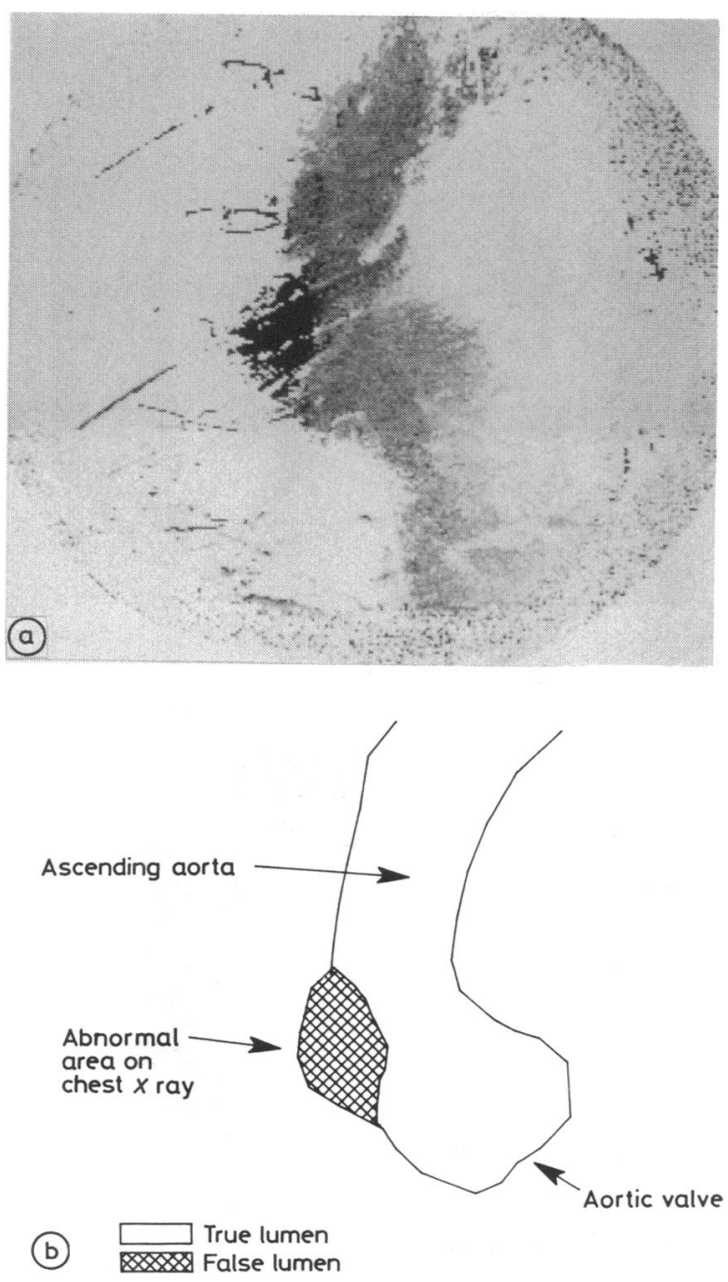

Fig 5 Arrival-time image (a) in a patient after operation. There is a small leak of contrast medium around the Dacron sleeve in the ascending aorta. (b) Explanatory diagram.

medium into a false lumen at the site of the proximal anastomosis (fig 5). This finding was subsequently confirmed by conventional cineaortography.

\section{Discussion}

The thoracic aorta is very suitable for investigation by intravenous digital angiography ${ }^{2}$ because it is relatively immobile and the images do not require a high degree of spatial resolution or contrast density. We found that digital subtraction was as accurate as cineaortography in the diagnosis of aortic dissection. ${ }^{1}$ But the possibility of mistaking a sub- traction artefact for an intimal dissection flap has led. to efforts to develop analysis methods that do not $\Rightarrow$ depend on image subtraction. One such technique is? arrival-time imaging. ${ }^{3}$

In pixels that are enhanced by the arrival of con $-\frac{\overline{0}}{\bar{c}}$. trast medium the shade of that pixel in the arrival- $\bar{\nabla}$ time image will be determined by the time taken for 0 contrast to reach that pixel and also by the time takencs for that pixel to be maximally enhanced by contrast. $\overrightarrow{0}$ Thus it is possible to distinguish two lumens even if contrast enters simultaneously, provided the rate of $\vec{\sigma}$ flow in the two lumens is different.

In pixels that are not enhanced by contrast there should be little variation between the maximum ande minimum values. But all pixels will vary slightly, in $\tilde{\text { N }}$ random fashion, and therefore the arrival-time of the $\mathscr{C}_{0}$ background structures should be random. In prac-o tice, however, random variation in the background is masked by the effect of $x$ ray scatter. $X$ ray scatter 7 varies with the overall density of the structure undero investigation; the greater the density the greater the scatter. Therefore as contrast medium arrives in the structure under study the degree of background scat $-\vec{\varphi}$ ter will increase. This will have the effect of reducing $\infty_{\infty}^{\infty}$ the background image density, and hence the pixel density. This means that background image pixelso will achieve maximal density very early in the study, and this area will be represented as having a very early arrival-time. It would be possible to prevent $\frac{}{\Phi}$ this effect by correcting the images for variation in $x \propto$ ray scatter. This is not necessary, however, as the $\overrightarrow{0}$ very early arrival-time of the background structures 3 enables them to be distinguished from the contrast enhanced structures.

The diagnosis of aortic dissection by arrival-time $\frac{\vec{\phi}}{\vec{\phi}}$ imaging of the thoracic aorta is dependent on the fact $\stackrel{9}{?}$ that contrast enhancement usually occurs later in the false lumen than in the true lumen. Thus these two $\frac{3}{\text {. }}$. lumens can be distinguished by a difference in arrival-time which is represented on the images as a difference in grey scale. This was found to be the case의 in four of the six patients with known aortic dis- $\rightarrow$ section, and in the patient in whom a residualo descending dissection was found after operation. N Furthermore, the site of entry of the dissection in the $e^{-}$ three patients with distal entry sites could be $\tilde{O}$ identified from the pattern of arrival-time throughout the false lumen. In two patients the subtraction images showed severe disruption of the intimal flape and no difference in flow could be seen between the $\bar{\varnothing}$ true and false lumens. It is therefore not surprising? that arrival-time imaging was unable to detect dissection in these patients. The arrival-time images $\frac{}{+}$ were normal in three patients in whom dissection had been excluded both by subtraction and by cine- $\frac{\mathbb{}}{2}$ aortography. 
In one case the arrival-time image helped in the interpretation of the subtraction aortogram. In this patient the subtraction images after operation were initially assessed as normal. When an arrival-time delay to the right-anterior aspect of the aortic root was demonstrated, however, it prompted reassessment of the subtraction images. A very faint image of contrast medium leaking into a false lumen was now seen. This illustrates another advantage of this technique-because the analysis is dependent on the maximal density of each pixel and not on the absolute pixel density, a minor degree of contrast enhancement will be detected to the same extent as considerable enhancement. Thus attention may be drawn to a false lumen which may not be noticed on the initial subtraction images.

Because analysis of arrival-time imaging throughout the whole time series is obviously subject to sideways motion it therefore has a lower spatial resolution than subtraction imaging. Sideways motion can also produce unexpected arrival-times at the edges of structures under study. So such images should be studied in conjunction with the subtraction images; thus a combination of the techniques may provide more information than either study alone.
In this series aortic dissection could readily be diagnosed from the subtraction images alone. In one patient, however, the diagnostic accuracy of the subtraction images was improved by the arrival-time image. In the other patients the arrival-time images confirmed the diagnosis. Thus this method of arrival-time analysis, when used in conjunction with the subtraction images, assists in the exclusion of subtraction artefact, and furthermore produces one static image of a whole time-series of images. We are studying a larger number of patients to determine whether arrival-time analysis should be recommended as a routine adjunct to digital subtraction aortography.

\section{References}

1 Lyons J, Gershlick A, Norell M, Rubens M, Magee P, Layton C. Intravenous digital subtraction angiography in the diagnosis and management of acute aortic dissection. Eur Heart $\mathcal{F}$ 1987;8:186-9.

2 Grossman LB, Buonocone E, Modic MT, Meaney TF. Digital subtraction angiography of the thoracic aorta. Radiology 1984;150:323-5.

3 Hunter GJ, Hunter JV, Brown NJ. Parametric imaging using digital subtraction angiography. $\mathrm{Br} \mathcal{F}$ Radiol 1986;59:7-11. 